\title{
Kinetics of Local Anesthetic Interactions with Model Membranes: Adsorption and Permeation
}

\author{
Gary Strichartz \\ Medical School, Boston, MA, USA \\ Email: gstrichartz@bwh.harvard.edu
}

Pain Research Center, Department of Anesthesiology, Perioperative and Pain Medicine, Brigham \& Women's Hospital, Harvard

How to cite this paper: Strichartz, G. (2022) Kinetics of Local Anesthetic Interactions with Model Membranes: Adsorption and Permeation. Open Journal of Anesthesiology, 12, 55-75.

https://doi.org/10.4236/ojanes.2022.121006

Received: November 5, 2021

Accepted: January 27, 2022

Published: January 30, 2022

Copyright $\odot 2022$ by author(s) and Scientific Research Publishing Inc. This work is licensed under the Creative Commons Attribution International License (CC BY 4.0).

http://creativecommons.org/licenses/by/4.0/

Open Access

\begin{abstract}
Introduction: Local anesthetics (LAs) must be adsorbed to and permeate through membranes to reach their sites of action. The rates of these processes critically affect drug actions at the cellular level and also the in vivo pharmacokinetics of perineurally injected drugs. Therefore, we measured the adsorption equilibria and permeation kinetics of two LAs local anesthetics with markedly different hydrophobicities: tetracaine (TTC, $\mathrm{P}_{\text {octanol:buffer }}=3.6 \times 10^{3}$ ) and procaine $\left(\mathrm{PRO}, \mathrm{P}_{\text {octanol:buffer }}=8.1 \times 10^{1}\right)$, in unilamellar membranes of different composition and fluidity. Methods: Interactions with unilamellar vesicles made of dipalmitoylphosphatidylcholine (DPPC), at $23^{\circ} \mathrm{C}$ (solid gel state) or $45^{\circ} \mathrm{C}$ (liquid crystal state), without or with cholesterol (Chol), were investigated by a combination of fluorescence and stopped-flow techniques. Membrane adsorption was monitored by the LA's fluorescence. Membrane permeation was monitored by the decrease of fluorescence intensity of intravesicular pyranine, when quenched by neighboring TTC or PRO. Results: Ionized TTC $\left(\mathrm{TTCH}^{+}\right)$and procaine $\left(\mathrm{PROH}^{+}\right)$adsorb to membranes more slowly and weakly than their neutral counterparts (time constant $\tau \sim 0.1 \mathrm{sec}$ ). Fluidizing of membranes, at the higher temperature $\left(45^{\circ} \mathrm{C}\right)$ or by Chol inclusion, accelerated LA adsorption (faster than the mixing time of the stoppedflow device, $0.008 \mathrm{~s}$ ). Permeation of protonated $\mathrm{TTCH}^{+}$through the solid gel DPPC membranes $\left(23^{\circ} \mathrm{C}\right)$ occurred far more slowly than adsorption, $(\tau=$ $36.7 \pm 0.7 \mathrm{~s}, \mathrm{n}=9)$, and 3 times slower than neutral TTC $(\tau=10.9 \pm 0.7 \mathrm{~s}, \mathrm{n}=$ 9); neutral PRO permeated these membranes at the same rate as TTC. Inclusion of Chol with DPPC, disordering the fatty acyl tails of membrane phospholipids while crowding their polar headgroups, slowed permeation of TTC and of PRO to an even greater degree. Conclusions: Local anesthetic permeation through membranes is limited by the transport across the membrane core and not by the initial binding. Drug ionization greatly slows permeation, but greater hydrophobicity does not facilitate it. Lipid crowding caused by
\end{abstract}


Chol, a normal membrane component, slows permeation by disorienting the

LA bound at the surface.

\section{Keywords}

Local Anesthetic, Fluorescence, Phospholipids, Kinetics, Stop-Flow

Technique, Adsorption, Permeation

\section{Introduction}

Many drugs interact with membranes in order to pass into cells to exert their intracellular effects or to penetrate to hydrophobic sites on membrane-associated channels and enzymes. Local anesthetics (LAs), class I antiarrhythmic agents, and other amphipathic agents exert their primary actions on ionic $\mathrm{Na}^{+}$channels from the cytosol after permeating the plasma membrane or from within the membrane phase itself [1] [2] [3] [4] [5]. Equally important may be the proposed role of the membrane in controlling the rate-limiting step for dissociation of the drug from its target protein, e.g. via the so-called "hydrophobic pathway" [6] [7].

Even more important for clinical applications, LAs must pass through epidural and perineural sheaths in order to reach the nerve fibers and block conduction [8] [9]. Delivery from the extraneural space, where injectate is deposited, to the intraneural compartment, is very inefficient, with only about $5 \%$ of the LA molecules in an injected $1 \%$ lidocaine solution $(37 \mathrm{mM})$ actually entering the nerve [10]. But the high concentration of injectate is essential for the effective block; in vivo studies in rats show that, for bolus injection, at least $1 \%-2 \%(37-75 \mathrm{mM})$ lidocaine is required for the transient conduction block of all fibers whereas at equilibrium (30 - $60 \mathrm{~min}$ exposure) only $1-2 \mathrm{mM}$ is fully effective [11] [12]. Therefore, improving the efficiency of LA membrane permeation would allow the use of lower concentrations and, possibly, reduce adverse side effects.

The equilibria of drug:membrane interactions have been extensively investigated previously using a variety of physical methods, including X-ray diffraction [13] [14], NMR [15] [16] [17] [18], ESR [19] [20] [21] and FTIR [22] [23] [24]. These methods, while providing good signal resolution, are far too slow to allow measures of the rates of membrane:drug interactions. Moreover, NMR usually requires a high concentration of the test substance, whereas high concentrations of tetracaine may significantly change a membrane's structure [16] [20] [25] [26].

To avoid these limitations we have previously used the intrinsic fluorescence of the LA tetracaine, at low concentrations, to study its equilibrium interactions with detergent micelles [27] [28], and lipid bilayer membranes [29]. In the present work, we have extended these equilibrium studies by examining the adsorption and permeation rates of the local anesthetics tetracaine and procaine with model membranes. Membranes formed as unilamellar vesicles were made 
of dipalmitoylphosphatidylcholine (DPPC), without or with cholesterol (27.5 mol\%), a normal component of plasma membranes, were used to answer the following questions: 1) How does LA hydrophobicity and ionization state affect membrane adsorption and permeation? 2) How does the molecular composition or physical state of the membrane (solid gel vs disordered) affect rates of LA adsorption into and permeation through a membrane?

\section{Materials and Methods}

Briefly, the adsorption rate was obtained from the increase in the LA's intrinsic fluorescence (at $350 \mathrm{~nm} / 302 \mathrm{~nm}$ excitation) with membrane binding [29]. The permeation rate of LA across the membrane was obtained from the decrease in fluorescence intensity $(509 \mathrm{~nm})$ of intravesicular pyranine, resulting from both direct interaction of intravesicular pyranine with LAs as they translocated into the intra-vesicular aqueous compartment, and from the $\mathrm{pH}$ rise caused by the basic LA's accumulation inside the vesicle [30] [31].

\subsection{Reagents}

Tetracaine $\cdot \mathrm{HCl}$, procaine $\cdot \mathrm{HCl}$ and pyranine were obtained from Sigma-Aldrich Chemicals (St. Louis, MO). DPPC was purchased from Avanti Polar Lipids (Alabaster, Alabama), was $>99 \%$ pure, and was stored at $-20^{\circ} \mathrm{C}$ in $\mathrm{CHCl}_{3}$ and used as received. Cholesterol was obtained from Avanti Polar Lipids (Alabaster, Alabama) as powder. Radiolabeled ${ }^{14} \mathrm{C}$-dipalmitoylphosphatidylcholine in toluene:methanol (1:1) was purchased from Perkin-Elmer Life and Analytical Sciences, Inc. (Boston, MA).

\subsection{Solutions}

All experiments were conducted in a standard aqueous medium (SAM), which contained $150 \mathrm{mM} \mathrm{NaCl}$ in doubly deionized (18 Mohm-cm), highly purified, pyrogen-free water (Water Systems 5601, Millipore Corp, Woburn, MA), buffered with a combination of 3 "Good" buffers, 2 mM CAPS [3-(cyclohexylamino)propanesulfonic acid, $\mathrm{pKa}=10.4$ ], $2 \mathrm{mM}$ BICINE [N,N-bis (2-hydroxyethyl) glycine, $\mathrm{pKa}=8.35$ ], and $2 \mathrm{mM}$ MES [2-(N-morpholino)-ethanesulfonic acid, $\mathrm{pKa}$ = 6.15] monohydrate (all obtained from Calbiochem-Behring Corp, La Jolla, $\mathrm{CA}$ ) in order to provide a constant buffering capacity over the broad $\mathrm{pH}$ range $5.5-11$.

\subsection{Membrane Preparation}

Large unilamellar vesicles (LUVETS), approximately $100 \mathrm{~nm}$ diameter, were created by the extrusion technique of Hope, et al. [32], using ${ }^{14} \mathrm{C}$-radiolabeled DPPC as a tracer to quantify yields and determine the concentration of LUVETS. Fifty milligrams of DPPC (an additional $10 \mathrm{mg}$ cholesterol in $\mathrm{CHCl}_{3}$ was added when making DPPC-Cholesterol LUVETS) and tracer were placed a round-bottom flask and vortexed together and the chloroform in which they were delivered was evaporated under $\mathrm{N}_{2}$ gas. $20 \mathrm{~mL}$ of SAM was then added to the lipid residue and 
the lipid-SAM mixture was heated in a water bath to $50^{\circ} \mathrm{C}-55^{\circ} \mathrm{C}$, a temperature above the solid gel-liquid crystal transition temperature $\left(41^{\circ} \mathrm{C}\right.$ for DPPC), and repeatedly vortexed until all lipid was in solution. A sample of the resulting mixture was counted on a Beckman Model LS 6500 liquid scintillation counter (10 $\mu \mathrm{L} / 5 \mathrm{ml}$ EcoLiteÔ liquid scintillation cocktail, ICN Biomedicals, Costa Mesa, $\mathrm{CA})$ to determine the specific radioactivity ( $\mathrm{cpm} / \mathrm{mmoles}$ lipid), the conversion factor to be used for calculation of lipid concentration.

The SAM-lipid mixture was alternately frozen (dry-ice/acetone bath) and thawed (in a $50^{\circ} \mathrm{C}-55^{\circ} \mathrm{C}$ water bath) at least five times to ensure complete hydration and equilibration (especially in the presence of cholesterol). The lipid mixture was then extruded, at $50^{\circ} \mathrm{C}$ at least ten times through a Northern Lipids (Vancouver, B.C.) extruder fitted with two $0.1 \mathrm{~mm}$ polycarbonate filters (BioRad, Westbury, NY) and driven by $\mathrm{N}_{2}$ gas at 400 psi, to produce LUVETS. A sample of this LUVET stock was counted by liquid scintillation to determine the concentration of phospholipid in the final membrane suspension. The LUVETS were stable overnight when stored at $4^{\circ} \mathrm{C}$.

To make pyranine-entrapping DPPC LUVET, a stock solution of $1.0 \mathrm{mM}$ pyranine was added to the initial lipid suspension to bring a desired pyranine concentration between the freezing/thaw and the extrusion steps. Unentrapped pyranine was removed by a slightly modified procedure of gel filtration chromatography [31]. Two milliliters of concentrated pyranine-entrapping DPPC LUVET suspension was placed on a gel filtration column (Sephadex G-25, medium grade) and eluted with SAM, using pyranine's fluorescence at $509 \mathrm{~nm}$ (455 nm excitation) to monitor the separation. The fractions without extra-vesicular pyranine were collected and the DPPC LUVET concentrations were determined by liquid scintillation counting. Repeated centrifugation and washing (x3) were applied to remove any residual extravesicular pyranine.

\subsection{Fluorescence Measurements}

Equilibrium distribution coefficients and partitioning coefficients for tetracaine and procaine were determined by the method of Zhang et al. [29]. To avoid light scattering caused by vesicles, front-face illumination was used with a triangular cuvette $\left(45^{\circ}, 45^{\circ}\right.$ and $\left.90^{\circ}\right)$. Fluorescence emission spectra were obtained with excitation at $302 \mathrm{~nm}$ using an Aminco spectrofluorometer (model SPF-500ä C) with a xenon lamp and direct temperature control of the cuvette (Aminco, Urbana, IL). Kinetic studies employed a stopped-flow (rapid mixing) device with equal volumes of LA solution and membranes delivered from $\mathrm{N}_{2}$ gas-driven syringes. Measurements of fluorescence changes due to simple dilution of LA solutions into buffer showed that mixing was complete after $0.008 \mathrm{sec}$. The computer collecting the fluorescence intensity data was programmed to begin collecting data after this time, identified as $\mathrm{t}=0$.

\subsection{Experimental Design}

Net membrane permeation of LA has three steps in sequence: 1) Adsorption to 
the membrane's external leaflet from the extravesicular solution; 2) Trans-membrane transport; 3) Desorption from the inner leaflet into the intra-vesicular aqueous compartment.

\subsubsection{Kinetics of TTC's Adsorption into Membrane Bilayer}

Kinetic data were collected by combination of the Aminco spectrofluorometer with RX2000 Rapid Kinetics Spectrometer Accessory (Applied Photophysics Ltd, Surry, United Kingdom), both of which were equipped with a water bath for temperature control. The LUVETs and LA solution were mixed at equal volume in the flow cell of the mixing accessory. The "dead-time" of this "stopped-flow" device, defined by the time until mixing was complete, was determined by simple dilution kinetics of tetracaine's fluorescence, and was $0.008 \mathrm{sec}$. All reported concentrations are post-mixing, unless otherwise stated.

The emission maxima of fluorescence, arising from the aromatic moiety of the LAs, is blue-shifted from $370 \mathrm{~nm}$ in aqueous solution to $350 \mathrm{~nm}$ in DPPC membrane bilayer and its intensity is greatly enhanced [27] [29]. Therefore, the rate of initial membrane adsorption of LA binding to the outer leaflet was monitored by the time course of the fluorescence increase at $350 \mathrm{~nm}$.

\subsubsection{Kinetics of LA Transmembrane Transport}

Pyranine is a highly water-soluble, $\mathrm{pH}$-sensitive fluorophore whose polarity is so high that it will remain trapped within the vesicles over several days [31] [33] [34]. In order to determine the role of drug ionization state on permeation rates experiments were conducted with both intra-vesicular and external solutions at either $\mathrm{pH}$ 5.5, for ionized LA, $\mathrm{TTCH}^{+}$and $\mathrm{PROH}^{+}$, or at $\mathrm{pH} 11$, for neutral TTC and PRO, respectively. We assume that inner and outer leaflets of the bilayer are symmetrical and thus that binding:dissociation rates of the LA at the inner leaflet occurs at the same, rapid rate as at the external leaflet. Therefore, the change in intra-vesicular pyranine fluorescence reflects the rate-limiting step for net membrane transport of $L A$.

\subsection{Statistical Analysis}

Partition coefficients were calculated as described in Zhang et al. [29]. Kinetic data were collected and averaged in each experiment from multiple $(n=8-10)$ mixing steps of the same LA:membrane conditions, to reduce noise and allow better curve fitting. Curves of exponential functions were fit to the data using Origin software (v. 6, Northampton MA), and the initial rates or extracted time-constants, $\tau$, from 3 - 5 separate experiments were averaged and reported as mean \pm SEM. Comparison by ANOVA of the $\tau$ values under different conditions are reported, with $\mathrm{p}<0.05$ considered significant.

\section{Results}

\section{Equilibrium Partitioning of Procaine}

The role of drug hydrophobicity is explored here by comparing moderately hy- 
drophobic procaine with its more highly hydrophobic homologue, tetracaine. Drug structures are shown in Figure 1, and Partition Coefficients between octanol and buffer and between membranes and buffer are listed in Table 1.

Procaine's membrane location. The fluorescence of LA's results from light emission from the excited $\mathrm{N}$-substituted aromatic region of the molecules (Figure 1). Fluorescence of neutral PRO (measured at $\mathrm{pH} 11.0$ ) was greatly enhanced and the emission fluorescence maximum (302 $\mathrm{nm}$ excitation) was blue-shifted, from $355 \mathrm{~nm}$ for aqueous PRO to $344 \mathrm{~nm}$ when bound to DPPC<smiles>CCN(CC)CCOC(=O)c1ccc(N)cc1</smiles>

procaine<smiles>CCCCNc1ccc(C(=O)OCCN(C)C)cc1</smiles>

tetracaine

Figure 1. Structures of procaine and tetracaine, in their unionized form. Fluorescence results from light emitted from the excited electrons of the aromatic regions. Note the difference in the molecular locations of the hydro-phobic alkyl substituents: 1 ) $-\mathrm{C}_{2} \mathrm{H}_{5}$ on the tertiary amine of PRO, $-\mathrm{CH}_{3}$ on TTC; 2 ) unsubstituted $-\mathrm{NH}$ on PRO but $-\mathrm{C}_{4} \mathrm{H}_{9}$ on the para-amino substituent of TTC.

Table 1. Partition coefficients of local anesthetics.

\begin{tabular}{|c|c|c|c|}
\hline Anesthetic & OCT:Buffer ${ }^{+}$ & DPPC:Buffer & $\mathrm{P}_{\mathrm{DPPC}}: \mathrm{P}_{\mathrm{OCT}}$ \\
\hline PRO & $8.1 \times 10^{1}$ & $4.3 \times 10^{3}$ & $5.3 \times 10^{1}$ \\
\hline $\mathrm{PROH}^{+}$ & $2.0 \times 10^{-3}$ & $1.7 \times 10^{3}$ & $8.5 \times 10^{2}$ \\
\hline TTC & $3.62 \times 10^{3}$ & $1.2 \times 10^{4} \star *$ & 3.3 \\
\hline $\mathrm{TTCH}^{+}$ & $4.6 \times 10^{-1}$ & $4.8 \times 10^{3 * *}$ & $1.04 \times 10^{4}$ \\
\hline $\mathrm{PRO} / \mathrm{PROH}^{+}$ & $4.0 \times 10^{4}$ & 2.5 & \\
\hline $\mathrm{TTC}^{\mathrm{TTCH}}{ }^{+}$ & $7.9 \times 10^{3}$ & 2.5 & \\
\hline TTC/PRO & $4.5 \times 10^{1}$ & 2.8 & \\
\hline $\mathrm{TTCH}^{+} / \mathrm{PROH}^{+}$ & $2.3 \times 10^{2}$ & 2.8 & \\
\hline
\end{tabular}

${ }^{*}$ Rate of mole fractions for LA-Ocranol and LA-DPPC systems. ${ }^{+}$From Strichartz et al. [30]. ${ }^{*}$ From Zhang et al. [29]. 
membranes (data not shown). The equivalent dielectric constant of membranebound PRO, determined by the maximum blue-shift calibrated by the same method of dioxane-water system used for TTC [29], was 10 - 15, far less than an aqueous solvent's (80) and slightly higher than that of membrane-bound neutral TTC, 6 - 10, implying that neutral PRO binds less deeply in the membrane than neutral TTC. However, for $\mathrm{PROH}^{+}$no fluorescence maxima shift and only a $20 \%$ increase in fluorescence intensity was observed upon membrane binding, suggesting that $\mathrm{PROH}^{+}$is located at an even more superficial location than PRO.

The membrane:buffer partition coefficient, expressed as: [molesPRO/molesDPPC]/ [molesPRO/moles $\mathrm{H}_{2} \mathrm{O}$ ], is 2.5 greater for $\mathrm{PRO}$ than $\mathrm{PROH}^{+}$, the same ratio occurs for unionized to ionized tetracaine, although tetracaine partitions to DPPC membranes 2.8 times more than procaine (Table 1 ). In other words, LA charge affects equilibrium adsorption to liquid crystal bilayers to the same extent, regardless of the difference in a molecule's hydrophobi city or location on the membrane's surface. Importantly, partition coefficients of LAs to membranes are 50 - 10,000 greater than for octanol, demonstrating how poor a system that solvent is for modelling membranes.

Kinetics of membrane adsorption of TTC.

Effect of Ionization on adsorption rates. Typical curves showing the increased fluorescence of tetracaine when rapidly mixed with DPPC membranes are shown in Figure 2. At time 0 the mixing of an equal volume of $2 \mathrm{mM} \mathrm{DPPC}$

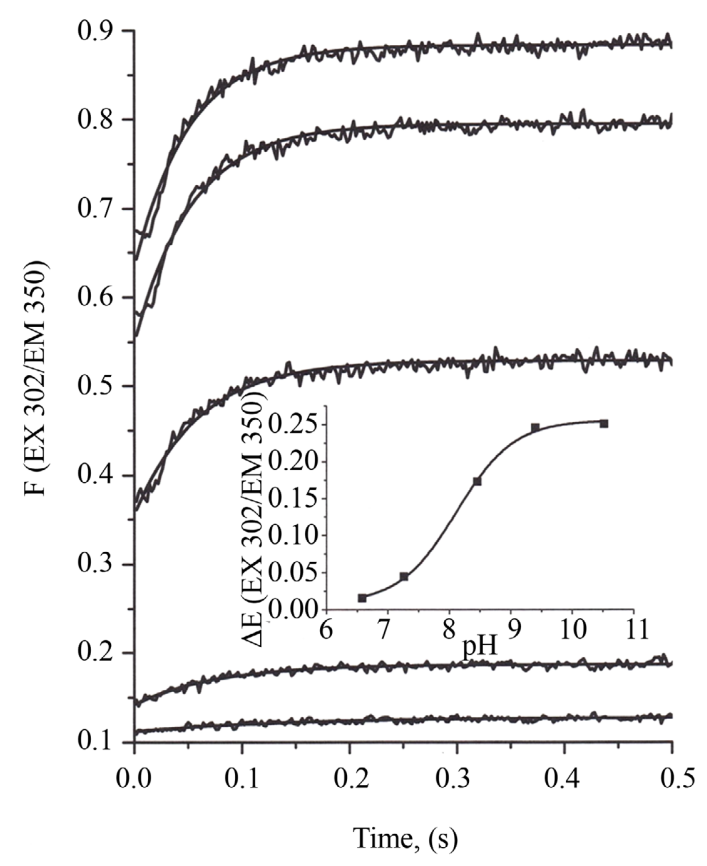

Figure 2. A typical set of time-courses of adsorption of $20 \mathrm{mM}$ TTC by $1 \mathrm{mM}$ DPPC LUVET at different $\mathrm{pH}$ values, $23^{\circ} \mathrm{C}$. $\mathrm{pH}$, from top-down, is $10.52,9.40,8.46,7.26$ and 6.58. Each curve is an average of at least 5 measurements. The insert plots fluorescence change amplitudes against $\mathrm{pH} ; \mathrm{pK}_{\mathrm{a}}=8.12 \pm 0.1$ was obtained by fitting the data using Boltzmann equation, DF $=\mathrm{F} 2+(\mathrm{F} 1-\mathrm{F} 2) /\left(1+\exp \left(\left(\mathrm{pH}-\mathrm{pK}_{\mathrm{a}}\right) / \mathrm{pH}\right)\right)$. 
LUVETs with $40 \mu \mathrm{M}$ TTC was complete and the fluorescence changes reflect the binding of tetracaine to the membrane, at $\mathrm{pH}$ values ranging from 10.5 (upper curve) to 6.6. The curves were fit by a single exponential function to extract time constant $(\tau)$ and amplitude changes (DF; see Methods). Both rates and steady-state changes (after $0.3 \mathrm{sec}$ ) increase with higher $\mathrm{pH}$, showing the faster and greater adsorption of the uncharged drug species. The plot of final DF against $\mathrm{pH}$ (inset, Figure 2) is fit with a $\mathrm{pK}_{\mathrm{a}}$ of $8.12 \pm 0.1$, in good agreement with the $\mathrm{pK}_{\mathrm{a}}$ of 8.17 for DDPC adsorption reported previously [29], and lower than the $\mathrm{pK}_{\mathrm{a}}$ of tetracaine in buffer, $8.59 \pm 0.04$ [30]. Time constants for a simple first-order process, such as adsorption: desorption, are related to the rate constants for those processes by Equation (1):

$$
\tau^{-1}=k_{\text {on }}[\mathrm{TTC}]+k_{\text {off }}
$$

where $k_{\text {on }}$ is the first-order rate constant for adsorption and $k_{\text {off }}$ the desorption rate constant. The plot of inverse time constant $\left(\tau^{-1}\right)$ versus uncharged TTC concentration is shown in Figure 3, based on $\tau$ values determined from fits to the data like those in Figure 1. Linear regress ion fit allows calculation of $k_{\text {on }}$, from the slope, and $k_{\text {off }}$ from the Y-axis intercept. The neutral TTC concentrations were calculated using a $\mathrm{pK}_{\mathrm{a}}$ value of aqueous tetracaine [30] and the free [TTC] $=20 \mathrm{mM}$. (The correlation coefficients of the fit, 0.87 and 0.89 , are the same ( $\mathrm{p}>0.10, t$-test) whether the $\mathrm{pK}_{\mathrm{a}}$ of aqueous or of membrane-bound tetracaine is used to determine charged and neutral concentrations.) A similar analysis for ionized $\mathrm{TTCH}^{+}$yields rate constants for the charged species. These rate constants are displayed in Table 2, along with the initial rates of adsorption determined by the initial slope of the traces in Figure 2. (The negative values of $k_{\text {on }}$ is real and probably results from the electrostatic repulsion of free $\mathrm{TTCH}^{+}$by the

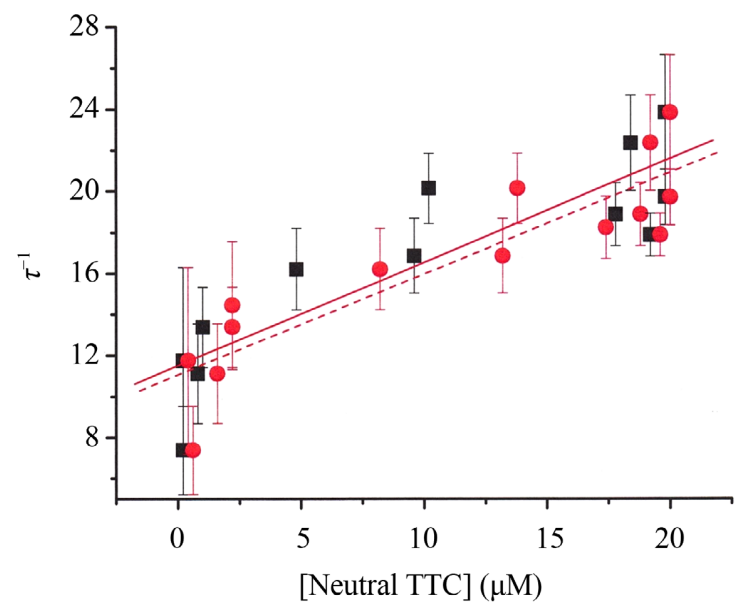

Figure 3. Plot of $\tau^{-1}$ versus neutral TTC concentration calculated by total concentration of $20 \mathrm{mM}$ multiplied by the percentage of neutral TTC at specific $\mathrm{pH}$ values. $\mathrm{n}$ represents neutral TTC concentrations based on the $\mathrm{pK}_{a}^{a}$ of aqueous free TTC, the equation, $\tau^{-1}=$ $11.5( \pm 1.16)+0.50( \pm 0.09)$ [neutral TTC] with linear coefficient 0.87$)$ was obtained from the linear fit of the data (solid line); - was obtained from the linear fit of the data (dashed line) based on the $\mathrm{pK}_{a}^{m}$ of DPPC membrane-bound TTC. 
Table 2. Rate constants of adsorption $\left(k_{\mathrm{on}}\right)$ and desorption $\left(k_{\mathrm{off}}\right)$ and Initial bindingrate constants for partitioning into DPPC membranes.

\begin{tabular}{cccc}
\hline $\mathrm{pH}$ & $k_{\text {on }}\left(\mathrm{M}^{-1} \cdot \mathrm{s}^{-1}\right)$ & $k_{\text {off }}\left(\mathrm{s}^{-1}\right)$ & $\begin{array}{c}\text { Initial binding } \\
\text { rate constants }\end{array}$ \\
\hline 11 & $2.3 \pm 1.8 \times 10^{4}(N=8)$ & $15.6 \pm 2.6(N=8)$ & $4.8 \pm 1.9 \times 10^{4}(N=8)$ \\
6.45 & $-2.1 \pm 0.7 \times 10^{5}(N=8)$ & $20.9 \pm 4.0(N=8)$ & $6.2 \pm 3.3 \times 10^{3}(N=8)$ \\
\hline
\end{tabular}

charged $\mathrm{TTCH}^{+}$bound to the membrane surface; see Discussion). For neutral TTC adsorption the calculated $k_{\text {on }}$ from fits to Figure 3 are the same as those from the initial slope ( $\mathrm{p}>0.05$ ), about $2-5 \times 10^{4} \mathrm{M}^{-1} \cdot \mathrm{s}^{-1}$. Therefore, at $20 \mu \mathrm{M}$ TTC the product of concentration and adsorption rate constant equals an adsorption rate of $0.4-1.0 \mathrm{~s}^{-1}$ compared to $\sim 16 \mathrm{~s}^{-1}$ for the desorption rate. Using the initial binding rate to estimate $k_{\text {on }}$ for $\mathrm{TTCH}^{+}$shows, by the same analysis, an adsorption rate of $0.012 \mathrm{~s}^{-1}$ and a desorption rate of $20 \mathrm{~s}^{-1}$. Thus, desorption of the neutral and charged species of tetracaine from DPPC membranes occur at about the same rate whereas adsorption of the charged species is about 8 -fold slower than that of the neutral form. But regardless of the ionization state of tetracaine, the desorption reaction is the dominant term controlling equilibrium partitioning.

Effect of temperature on adsorption rates. In order to study the effect of membrane phase, experiments were also conducted at $45^{\circ} \mathrm{C}$, where the membranes are in the liquid-crystal state rather than the solid-gel phase that exists at $23^{\circ} \mathrm{C}$ (the phase transition temperature for DPPC membranes is $41^{\circ} \mathrm{C}$ ). However, we found that the membrane binding of TTC at this higher temperature was too fast to be measured. Figure 4 shows the fluorescence intensity for $\mathrm{TTCH}^{+}$ ( $40 \mu \mathrm{M}, \mathrm{pH}=5.97$ ) after mixing with $2 \mathrm{mM} \mathrm{DPPC}$, at $45^{\circ} \mathrm{C}$ (open squares). Fluorescence traces for dilution of $40 \mu \mathrm{M}$ TTC in buffer (stars) and for $2 \mathrm{mM} \mathrm{DPPC}$ mixed with buffer (open circles) are also shown, along with the equilibrium spectra of those mixtures after 10 mins (inset). The enhanced intensity and blue shift of TTC plus membranes (inset) shows that adsorption did occur, but too rapidly to be detected (mixing time $=0.008 \mathrm{sec}$ ). Therefore, the adsorption of tetracaine to liquid-crystal membranes is much faster than adsorption to solid-gel membranes (e.g., Figure 2), paralleling the larger partition coefficient in the liquid crystal [29].

Inclusion of cholesterol in DPPC membrane accelerates membrane binding. Cholesterol's ability to reduce the mobility of phospholipids in liquid crystal membranes both by increasing hydrocarbon chain ordering and increasing membrane thickness of bilayers is well documented [35] [36] [37] [38] [39]. Inclusion of cholesterol in pure DPPC membranes in the solid-gel state at $23^{\circ} \mathrm{C}$ also changes the dynamics of the lipids, disordering the otherwise tightly packed acyl chains and causing steric crowding near the polar headgroups [38] [39]. We found that the adsorption of tetracaine into DPPC-Chol membranes was too fast to be detectable both at $23^{\circ} \mathrm{C}$ and $45^{\circ} \mathrm{C}$ (data not shown). Apparently, the 


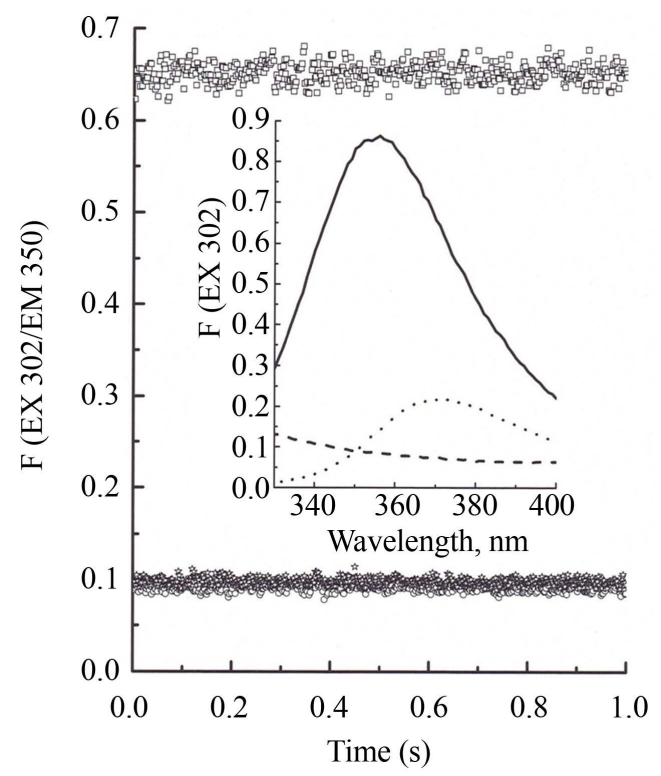

Figure 4. Time course curves of adsorption of total $20 \mathrm{mM}$ TTC by equal volume of 1 mM DPPC LUVET ( $\square$ ), mixing of $2 \mathrm{mM}$ DPPC LUVET with SAM ( $\bigcirc$ ), mixing of 40 mM TTC with SAM (y), respectively, at $\mathrm{pH} 5.97$ and $45^{\circ} \mathrm{C}$. Inserted are steady-state fluorescence emission spectra from the mixture composed of $20 \mathrm{mM}$ TTC and $1 \mathrm{mM}$ DPPC LUVET (solid), $1 \mathrm{mM}$ DPPC LUVET (dashed) and $20 \mathrm{mM}$ TTC (dotted), respectively, at $\mathrm{pH} 5.97$ and $45^{\circ} \mathrm{C}$.

loosening of tight packing of the phospholipid acyl chains, by the addition of cholesterol or by the change in phase at high temperature, both increase the adsorption to undetectably fast rates. Since, as noted above, the time constant for the membrane binding reaction is dominated by the desorption rate, the now undetectably fast rate means that the desorption rate is much greater than in the Chol stiffened membranes, an observation consistent with the reduction of TTC's equilibrium partitioning by Chol inclusion [29]. Tetracaine does not bind as tightly to membranes when $\mathrm{Chol}$ is present because it dissociates from the membrane more rapidly.

\section{Local anesthetic permeation through membrane.}

Interaction of pyranine with TTC. As shown in Figure 5, the fluorescence of pyranine in solution $(20 \mu \mathrm{M})$ was progressively reduced at increasing concentrations of TTC. The maximum inhibition was reached at a TTC concentration of about $40 \mathrm{mM}$ (inset, Figure 5), allowingdynamic response to TTC at the concentrations studied here. Similar inhibitions of pyranine fluorescence were observed at other experimental conditions, e.g., $\mathrm{pH} 11$ and $45^{\circ} \mathrm{C}$, and $\mathrm{pH} 7.4$ and $23^{\circ} \mathrm{C}$. A control kinetic experiment with TTC added to pyranine showed that the inter action was complete within $8 \mathrm{~ms}$, the dead time of the mixing accessory, which is much faster than the permeation time constants (shown below) and will not limit their measurement.

Effect of ionization of TTC on permeation through solid-gel membrane.

Permeation of tetracaine was monitored by changes in intravesicular pyranine, which does not pass across the membrane [30] [31]. Typical curves of 


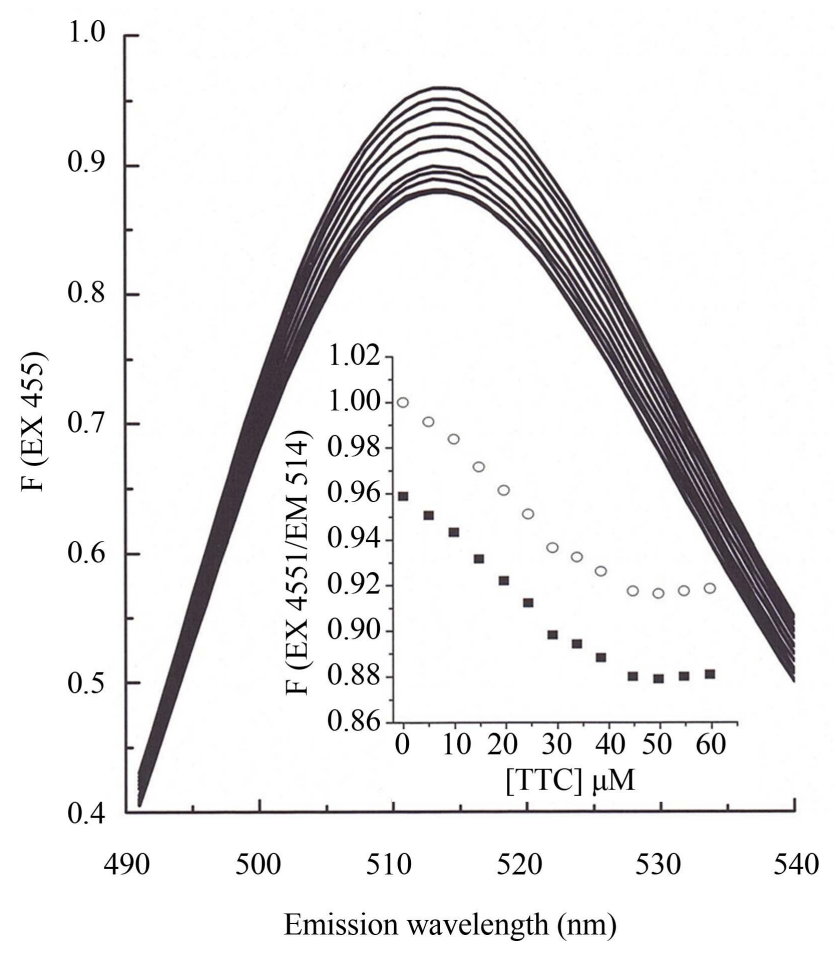

Figure 5. Steady-state fluorescence spectra of $2 \mathrm{~mL}$ of $20 \mu \mathrm{M}$ pyranine in buffer (the top curve at peak emission) with successive titration of $10 \mu \mathrm{L}$ of $1 \mathrm{mM}$ TTC, at $\mathrm{pH} 11$ and $23^{\circ} \mathrm{C}$. The curves were plotted after volume correction. The insert shows peak emission at $514 \mathrm{~nm}$ versus TTC concentration: - represents measured data, 0 represents normalized data by setting the peak intensity without TTC equal to 1 .

pyranine fluorescence after addition of TTC to DPPC LUVETS are shown in Figure 6 , for a range of $\mathrm{pHs}$, at $23^{\circ} \mathrm{C}$. In every case, the intra-vesicular and external solution were at the same initial $\mathrm{pH}$. The initial difference in pyranine fluorescence (at $t=0$ ) is due to its inherent $\mathrm{pH}$-dependence, but the subsequent drop in fluorescence reflects the rate at which TTC enters the intra-vesicular space, faster at higher $\mathrm{pHs}$ than at lower $\mathrm{pHs}$, suggesting that the neutral species permeates these solid-gel DPPC membranes more rapidly than the charged species. The curves were fitted with single exponential functions to extract a permeation time constant $(\tau)$. A graph of $\tau^{-1}$ against the concentration of neutral tetracaine, calculated for the different $\mathrm{pH}$ values used in Figure 6 at $20 \mu \mathrm{M}$ TTC, is shown in Figure 7. The slowest rate, with $\tau^{-1}=0.028 \pm 0.01 \mathrm{~s}^{-1}$, occurs for ionized $\mathrm{TTCH}^{+}$, at $\mathrm{pH}$ values less than 6.5; rates increase with increasing fraction of TTC in the neutral form. Note that the rates for permeation, indicated by these inverse time constants, are more than 100 times smaller than those for membrane adsorption (Figure 3).

The rate constants of TTC permeation in and out the DPPC bilayer at $23^{\circ} \mathrm{C}$ can be obtained from the slopes of plots of $\tau^{-1} v s$ TTC concentration (cf. Equation (1), above) and are collected in Table 3. Negative values of $k_{\text {in }}$ represent the downward curve of permeation rate with concentration in Figure 7, suggesting that the permeation process reaches a rate-limiting step at relatively low TTC 


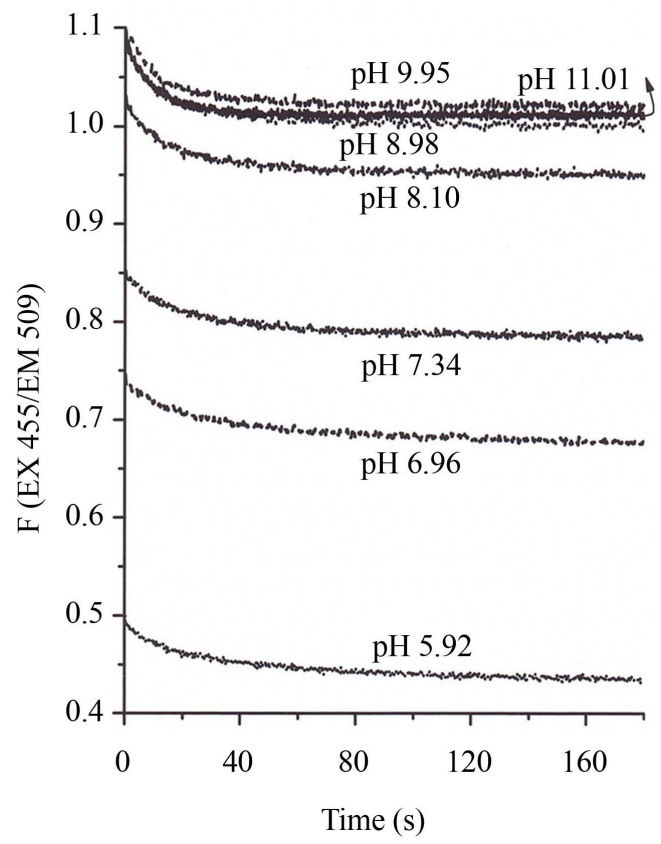

Figure 6. Time course curves of permeation of $20 \mu \mathrm{M}$ TTC through $1 \mathrm{mM}$ DPPC LUVET at $23^{\circ} \mathrm{C}$ and different $\mathrm{pH}$ values. Each curve was averaged from at least 5 measurements.

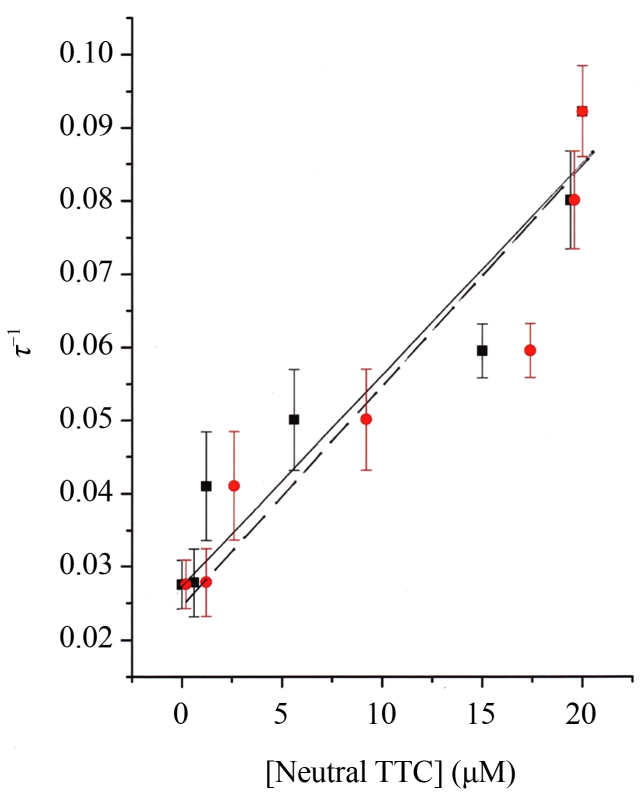

Figure 7. Inverse time constant of the permeation rate vs concentration of neutral TTC, determined by $\mathrm{pH}$ (total tetracaine concentration equals $20 \mu \mathrm{M}$ ). nrepresents data with [TTC] using the aqueous $\mathrm{pKa}, \bullet$ represents [TTC] using the membrane-bound pKa.

Table 3. Rate constants of TTC permeation through membrane at $23^{\circ} \mathrm{C}$.

\begin{tabular}{ccc}
\hline $\mathrm{pH}$ & $k_{\text {in }}\left(\mathrm{M}^{-1} \cdot \mathrm{s}^{-1}\right)$ & $k_{\text {out }}\left(\mathrm{s}^{-1}\right)$ \\
\hline 11 & $-1.35( \pm 1.29, N=8) \times 10^{2}$ & $0.06517 \pm 0.00327(N=8)$ \\
5.91 & $-1.08( \pm 0.79, N=8) \times 10^{2}$ & $0.03147 \pm 0.002(N=8)$ \\
\hline
\end{tabular}


concentrations. The reverse rate constant, $k_{\text {out }}$, at $\mathrm{pH} 11$ is twice the value at $\mathrm{pH}$ 5.9 showing that the neutral species of TTC has a net permeation twice as fast as the charged species.

Inclusion of cholesterol slows TTC s permeation through solid-gel membranes. The effect of cholesterol on tetracaine's permeation through DPPC membranes at pH 9 is shown in Figure 8(a). Permeation through the DPPC-Chol membrane is more than 10-times slower than that through the DPPC membrane. Figure 8(b) shows pyranine fluorescence time course for TTC permeation through DPPCChol membrane at $23^{\circ} \mathrm{C}$ at different $\mathrm{pHs}$. (Note the difference in the $\mathrm{Y}$ axis scale with Figure 8(b)). No significant fluorescence signal decays were observed at $\mathrm{pH}$ 5.98 to 10.7 , showing that cholesterol inclusion greatly slows permeation for both charged and neutral tetracaine species.

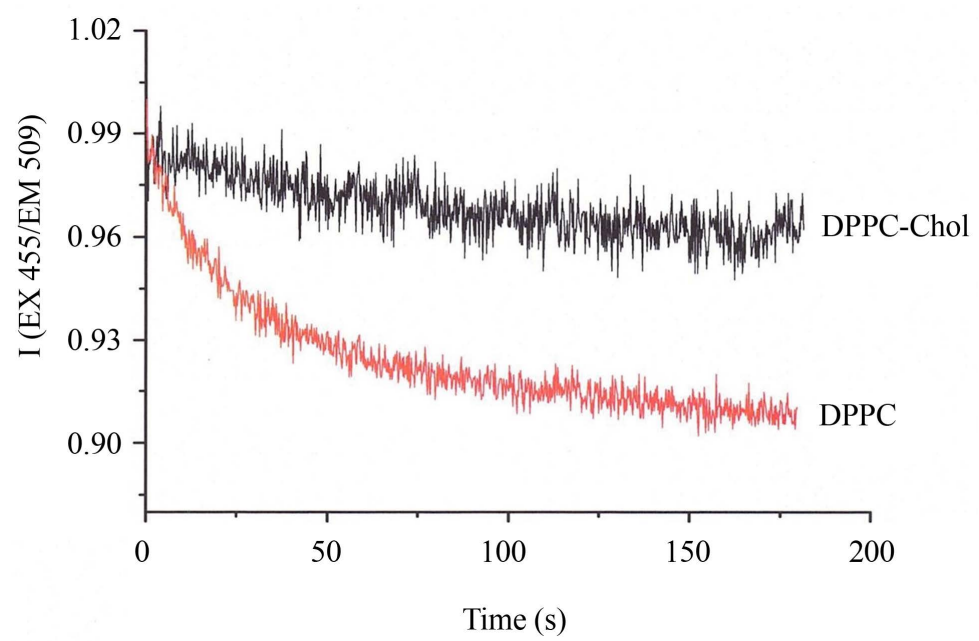

(a)

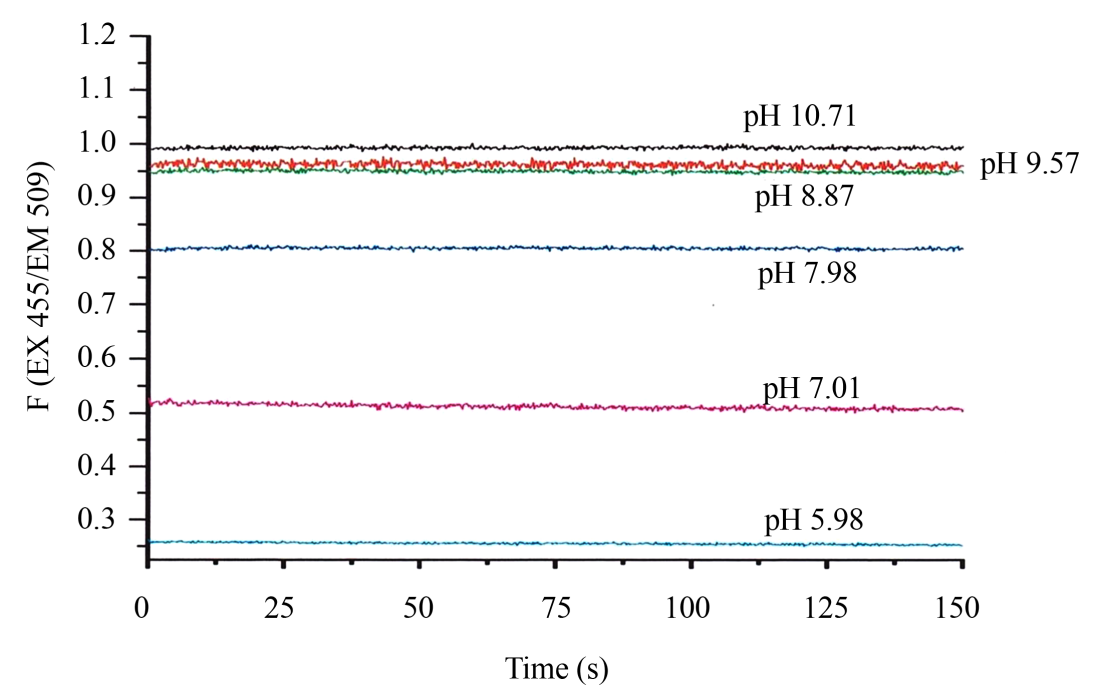

(b)

Figure 8. Time-course of permeation of $20 \mu \mathrm{M}$ TTC through (a) DPPC and DPPC-Chol membranes, $23^{\circ} \mathrm{C}, \mathrm{pH} 7.0$; (b) Time-course of permeation of $20 \mu \mathrm{M}$ TTC through DPPC-Chol membranes at different $\mathrm{pH}$ values. 
Comparison of TTC and PRO permeation through solid-gel membranes.

The permeation time-courses for tetracaine and procaine through DPPC solid-gel membranes $\left(23^{\circ} \mathrm{C}\right)$ at physiological $\mathrm{pH}$ (7.4) are shown in Figure 9(a). Permeation of the two LAs occur at virtually identical rates; single exponential fitting to data gave time constants of 58 and $60 \mathrm{~s}$, at $40 \mu \mathrm{M}$ LA. Interestingly, cholesterol inclusion slowed PRO's permeation about 16-fold, 4-times more of an effect than that experienced by TTC, which was slowed 4.4-fold (Figure 9(b)). The crowding of DPPC phospholipids by cholesterol appears to have a greater effect on the transmembrane transport of more superficially bound procaine than on more deeply bound tetracaine.

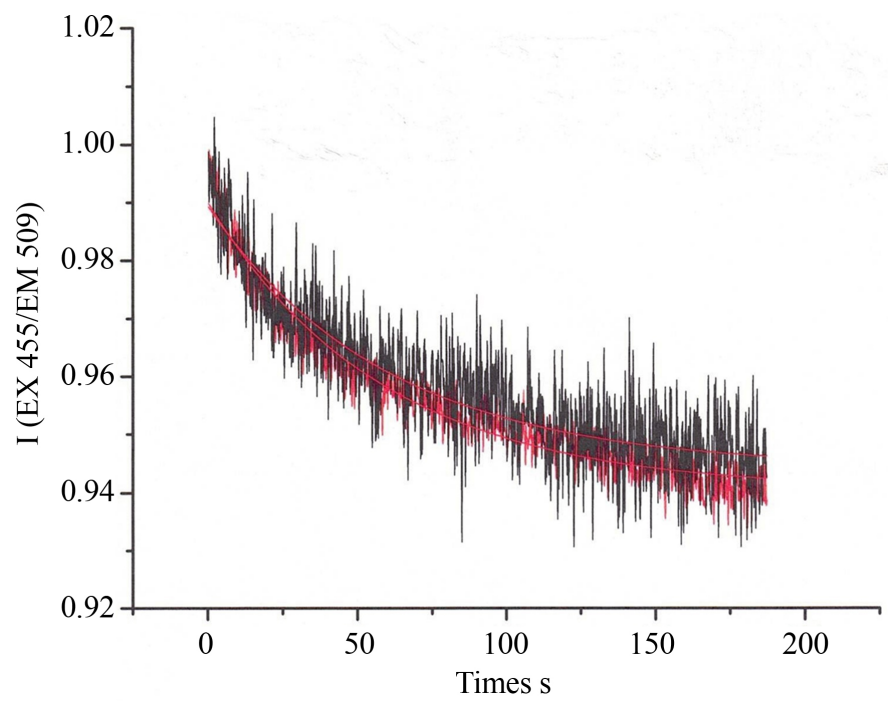

(a)

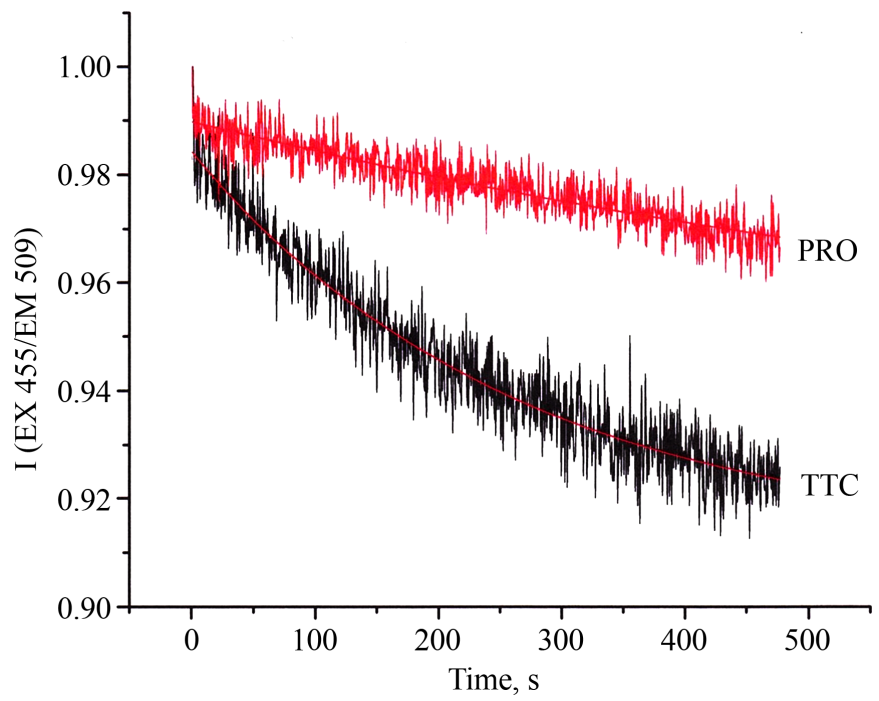

(b)

Figure 9. Time-courses for $40 \mu \mathrm{M}$ TTC (black trace) and $40 \mu \mathrm{M}$ PRO (red) permeation through (a) DPPC membranes and (b) DPPC + Chol membranes. Curves are fits to the data of single exponentials with (a) $\tau=58.2 \pm 1.4 \mathrm{~s}(N=20)$ for TTC and $60.0 \pm 3.4 \mathrm{~s}(N$ =4) for PRO; (b) $\tau=264 \pm 12 \mathrm{~s}(N=5)$ TTC and $971 \pm 21 \mathrm{~s}(N=17)$ PRO. 


\section{Discussion}

Local anesthetic molecules interact with membranes in order to cross them and reach intracellular sites and to reach hydrophobic loci on membrane-intrinsic proteins. Local anesthetics are not freely dissolved in membranes but are predominantly adsorbed near the membrane's surface, with the ionizable amine group facing towards the aqueous (extracellular or intracellular) compartment and the aromatic moiety directed towards the membranes apolar hydrocarbon core (Figure 1). Ionic interactions between charged amino groups and polar head groups of phospholipids, dipole-dipole interactions between the LA ester or amide bind and the glycerol-lipid ester bonds of the membrane lipids, and hydrophobic interactions between the LA's aromatic regions and the acyl side chains of the phospholipids all contribute to the energetics of binding to and transport across the membrane.

We have found that the homologous LAs tetracaine and procaine bind rapidly and reversibly to simple bilayer membranes and are transported across them at a much slower rate. The unionized form of each LA partitions 2.5-times that of the ionized form, and more hydrophobic tetracaine partitions 2.8-times more than procaine, comparing both ionized and ionized species. Shifts in the fluorescence emission spectra upon adsorption to the membranes indicate that neutral and protonated tetracaine are in the same region of low dielectric constant, close to that of the phospholipid acyl chains (16 carbons for DPPC), despite the effect of ionization on partitioning. In contrast, procaine, whose neutral form appears to reside more superficially than TTC's, perhaps close to the glycerol ester bonds of DPPC, is located even further onto the surface of the membrane when ionized.

Rates for tetracaine's partitioning to solid-gel membranes are about 8 times faster for the neutral than the ionized species, reflected in a slower adsorption rate for the charged species. Rates for tetracaine's adsorption to liquid-crystal membranes are too fast to be measured, for either ionization state, suggesting that the relative immobility of the lipids in the solid gel slows the desorption process and tends to stabilize the LA on the membrane. Inclusion of cholesterol into the membrane also speeds the adsorption process to undetectable rates, perhaps due to the crowding of phospholipids in the head group region and the resultant weaker adsorption energy, consistent with the weaker equilibrium partitioning caused by cholesterol [29].

These findings are consistent with published studies of equilibrium binding of these LAs to membranes of various lipid composition. Tetracaine has been shown by NMR and X-ray diffraction to reside at a deeper position within the membranes [40] [41] [42] [43], and to be able to shift rapidly between multiple binding loci [42], and procaine to be located at a more superficial site [40] [42].

The rates of permeation of tetracaine and procaine through membranes are also responsive to drug ionization and membrane state. Permeation occurs far more slowly than the equilibration reaction of partitioning, so the rate-limiting step cannot be membrane binding. Neutral species of these LAs permeate more 
rapidly than charged species because of the high energy required for a charged form to pass into and through the core hydrocarbon with its very low dielectric constant. Despite their different hydrophobicities and equilibrium partitioning, tetracaine and procaine permeate pure DPPC (solid-gel) membranes at the same rate. Lower partitioning of procaine corresponds to a weaker binding energy in the membrane and, perhaps a lower activation energy to cross the membrane's hydrocarbon core, whereas the higher partitioning of tetracaine will concentrate more drug on the membrane, and at a deeper location from which it may experience a lower energy in crossing the core. Inclusion of cholesterol, which is a common component of all mammalian plasma membranes, lowers partitioning and slows permeation of both LAs, but of procaine more than tetracaine. Cholesterol's crowding of the polar head groups in phospholipid membranes reduces lateral mobility and thus may slow LA penetration between DPPC molecules, at a locus which is more populated by procaine than by tetracaine molecules. But a reduction in acyl chain rotational mobility also occurs with cholesterol inclusion and may also contribute to the reduced transport.

It is essential for an understanding of LA interactions with membranes to realize that the membrane is itself directly affected by the bound drug. At the very low concentrations $(20 \mu \mathrm{M})$ used in this study, tetracaine equilibrium partitioning results in a ratio of 1 TTC molecule per 250 DPPC molecules, and 1 $\mathrm{TTCH}^{+}$molecule per 580 DPPC molecules (see Equation (1), Results). At the much higher concentrations needed for conduction block in exposed nerves, about $1 \mathrm{mM}$ [11], each TTC molecule is present for every 5 - 10 lipid molecules. Membrane lipid fluidity, the rotational mobility and lateral diffusion of lipid molecules, are altered by the presence of LAs [23] [43] [44]. The dynamic exchange of lipids between the liquid crystal and solid gel domains that occurs in membranes, particularly real biological membranes composed of many different phospholipids and cholesterol, is shifted towards the fluid phase by the adsorbed LAs [44] [45] [46] [47], changing the structure and activity of membrane-intrinsic proteins such as ion transporters and pumps. In addition, the positive charge on ionized LAs is attracted to negative surface potential of membranes due to anionic phospholipids, and LA adsorption at the surface lowers that potential and thus alters the local concentration of charged molecules in solution [48] [49] [50]. Through these different mechanisms bound LAs will reduce their own membrane affinity; with increasing concentrations greater energy is required for subsequent binding, leading to a down-turning curve in the concentration dependence of LA binding. Such behavior likely accounts for the negative association rate constants observed in the tetracaine binding experiments reported here.

\section{Conclusion}

In summary, local anesthetics bind rapidly and reversibly to membranes at their outer surface and are transported more slowly across the hydrocarbon core. Drug ionization lowers binding to membranes of neutral phospholipids and 
drug hydrophobicity raises that binding; ionization slows drug permeation but greater hydrophobicity does not affect it. The fluidity of membranes, characterized by a dynamic fluctuation between solid-gel and liquid-crystal domains, favors LA binding and permeation and itself is shifted towards the more fluid behavior by the adsorbed LA molecules.

\section{Acknowledgements}

All experiments reported here and much of the analysis was conducted by Dr. Zhingzhong Zhang, The author was not able to contact Dr. Zhang, who therefore could not approve of its content but who unquestionably would have been included as an author.

\section{Funding}

Research supported by USPHS grant R01-GM064792. The secretarial support of Ms. Ellen Jacobson is greatly acknowledged.

\section{Conflicts of Interest}

The author declares no conflicts of interest regarding the publication of this paper.

\section{References}

[1] Narahashi, T., Frazier, T. and Yamada, M. (1970) The Site of Action and Active Form of Local Anesthetics. I. Theory and $\mathrm{pH}$ Experiments with Tertiary Compounds. Journal of Pharmacology and Experimental Therapeutics, 171, 32-44.

[2] Strichartz, G.R. (1973) The Inhibition of Sodium Channels in Myelinated Nerve by Quaternary Derivatives of Lidocaine. Journal of General Physiology, 62, 37-57. https://doi.org/10.1085/jgp.62.1.37

[3] Hille, B. (1977) The pH-Dependent Rate of Action of Local Anesthetics on the Node of Ranvier. Journal of General Physiology, 60, 475-496. https://doi.org/10.1085/jgp.69.4.475

[4] Herbette, L., Scarpa, A., Blasie, J.K., Wang, C.T., Hymel, L., Seelig, J. and Fleischer, S. (1983) The Determination of the Separate $\mathrm{Ca}^{2+}$ Pump Protein and Phospholipid Profile Structures within Reconstituted Sarcoplasmic Reticulum Membranes via X-Ray and Neutron Diffraction. Biochimica et Biophysica Acta, 730, 369-378. https://doi.org/10.1016/0005-2736(83)90354-1

[5] Wang, G.K., Russell, C. and Wang, S.Y. (2004) State-Dependent Block of VoltageGated $\mathrm{Na}^{+}$Channels by Amitriptyline via the Local Anesthetic Receptor and Its Implication for Neuropathic Pain. Pain, 110, 166-174. https://doi.org/10.1016/j.pain.2004.03.018

[6] Hille, B. (1997) Local Anesthetics: Hydrophilic and Hydrophobic Pathways for the Drug-Receptor Interaction. Journal of General Physiology, 69, 497-575. https://doi.org/10.1085/jgp.69.4.497

[7] Courtney, K. and Strichartz, G. (1984) Structural Elements Which Determine Local Anesthetic Activity. In: Strichartz, G.R., Ed., Handbook of Experimental Pharmacology. Local Anesthetics, Vol. 81, Springer-Verlag, Berlin, 53-94. https://doi.org/10.1007/978-3-642-71110-7_3 
[8] Sinnott, C., Cogswell, L.P., Johnson, A. and Strichartz, G.R. (2003) On the Mechanism by Which Epinephrine Potentiates Lidocaine's Peripheral Nerve Block. Anesthesiology, 98, 181-188. https://doi.org/10.1097/00000542-200301000-00028

[9] Leeson, S. and Strichartz, G. (2013) Kinetics of Uptake and Washout of Lidocaine in Rat Sciatic Nerve in Vitro. Anesthesia \& Analgesia, 116, 694-702. https://doi.org/10.1213/ANE.0b013e31827aed25

[10] Popitz-Bergez, F.A., Leeson, S., Strichartz, G.R. and Thalhammer, J.G. (1995) Relation between Functional Deficit and Intraneural Local Anesthetic during Peripheral Nerve Block: A Study in the Rat Sciatic Nerve. Anesthesiology, 83, 583-592. https://doi.org/10.1097/00000542-199509000-00018

[11] Huang, J.H., Thalhammer, J.G., Raymond, S.A. and Strichartz, G.R. (1997) Susceptibility to Lidocaine of Impulses in Different Somatosensory Afferent Fibers of Rat Sciatic Nerve. The Journal of Pharmacology and Experimental Therapeutics, 282, 802-811.

[12] Gokin, A.P., Philip, B. and Strichartz, G.R. (2001) Preferential Block of Small Myelinated Sensory and Motor Fibers by Lidocaine: In Vivo Electrophysiology in the Rat Sciatic Nerve. Anesthesiology, 95, 1441-1454.

https://doi.org/10.1097/00000542-200112000-00025

[13] Herbette, L.G., Mason, P.E., Sweeney, K.R., Trumbore, M.W. and Mason, R.P. (1994) Favorable Amphiphilicity of Nimodipine Facilitates Its Interactions with Brain Membranes. Neuropharmacology, 33, 241-249. https://doi.org/10.1016/0028-3908(94)90015-9

[14] Barcelo, F., Prades, J., Funari, S.S., Frau, J., Alemany, R. and Escriba, P.V. (2004) The Hypotensive Drug 2-Hydroxyoleic Acid Modifies the Structural Properties of Model Membranes. Molecular Membrane Biology, 21, 261-268. https://doi.org/10.1080/09687680410001716835

[15] Kelusky, E.C. and Smith, I.C. (1984) An ${ }^{2}$ H NMR Study of the Binding of Specifically Deuterated Tetracaine and Procaine to Phosphatidylcholine. Canadian Journal of Biochemistry and Cell Biology, 62, 178-184. https://doi.org/10.1139/o84-025

[16] Auger, M., Jarrell, H.C. and Smith, I.C. (1988) Interactions of the Local Anesthetic Tetracaine with Membranes Entrapping Phosphatidylcholine and Cholesterol: A $2 \mathrm{H}$ NMR Study. Biochemistry, 27, 4660-4667. https://doi.org/10.1021/bi00413a012

[17] Auger, M., Smith, I.C., Mantsch, H.H. and Wong, P.T. (1990) High-Pressure Infrared Study of Phosphatidylserine Bilayers and Their Interactions with the Local Anesthetic Tetracaine. Biochemistry, 29, 2008-2015. https://doi.org/10.1021/bi00460a008

[18] Kelusky, E.C. and Smith, I.C. (1983) Characterization of the Binding of the Local Anesthetics Procaine and Tetracaine to Model Membranes of Phosphatidylethanolamine: A Deuterium Nuclear Magnetic Resonance Study. Biochemistry, 22, 6011 6017. https://doi.org/10.1021/bi00294a049

[19] Noji, S., Takahashi, T. and Kon, H. (1982) A Spin-Label Study of the Correlation between Stomatocyte Formation and Membrane Fluidization of Erythrocytes. Biochemical Pharmacology, 31, 3173-3180. https://doi.org/10.1016/0006-2952(82)90546-9

[20] Frezzatti, W.A., Toselli, W.R. and Schreier, S. (1986) Spin Label Study of Local Anesthetic-Lipid Membrane Interactions. Phase Separation of the Uncharged Form and Bilayer Micellization by the Charged Form of Tetracaine. Biochimica et Biophysica Acta, 860, 531-538. https://doi.org/10.1016/0005-2736(86)90550-X

[21] Bianconi, M.L., do Amaral, A.T. and Schreier, S. (1988) Use of Membrane Spin La- 
bel Spectra to Monitor Rates of Reaction of Partitioning Compounds: Hydrolysis of a Local Anesthetic Analog. Biochemical and Biophysical Research Communications, 152, 344-350. https://doi.org/10.1016/S0006-291X(88)80720-4

[22] Auger, M., Jarrell, H.C., Smith, I.C., Wong, P.T., Siminovitch, D.J. and Mantsch, H.H. (1987) Pressure-Induced Exclusion of a Local Anesthetic from Model and Nerve Membranes. Biochemistry, 26, 8513-8516.

https://doi.org/10.1021/bi00400a003

[23] Auger, M., Jarrell, H.C., Smith, I.C., Siminovitch, D.J., Mantsch, H.H. and Wong, P.T. (1988) Effects of the Local Anesthetic Tetracaine on the Structural and Dynamic Properties of Lipids in Model Membranes: A High-Pressure Fourier Transform Infrared Study. Biochemistry, 27, 6086-6093.

https://doi.org/10.1021/bi00416a038

[24] Shibata, A., Ikawa, K. and Terada, H. (1995) Site of Action of the Local Anesthetic Tetracaine in a Phosphatidylcholine Bilayer with Incorporated Cardiolipin. Biophysical Journal, 69, 470-477. https://doi.org/10.1016/S0006-3495(95)79920-9

[25] Racanský, V., Béderová, E. and Pisková, L. (1988) The Influence of Local Anesthetics on the Gel-Liquid Crystal Phase Transition in Model Dipalmitoylphosphatidylcholine Membranes. General Physiology and Biophysics, 7, 217-221.

[26] Hata, T., Matsuki, H. and Kaneshina, S. (2000) Effect of Local Anesthetics on the Bilayer Membrane of Dipalmitoylphosphatidylcholine: Interdigitation of Lipid Bilayer and Vesicle-Micelle Transition. Biophysical Chemistry, 87, 25-36. https://doi.org/10.1016/S0301-4622(00)00175-7

[27] García-Soto, J. and Fernández, M.S. (1983) The Effect of Neutral and Charged Micelles on the Acid-Base Dissociation of the Local Anesthetic Tetracaine. Biochimica et Biophysica Acta, 731, 275-281. https://doi.org/10.1016/0005-2736(83)90019-6

[28] Desai, S., Hadlock, T., Messam, C., Chafetz, R. and Strichartz, G. (1994) Ionization and Adsorption of a Series of Local Anesthetics in Detergent Micelles: Studies of Drug Fluorescence. Journal of Pharmacology and Experimental Therapeutics, 271, 220-228.

[29] Zhang, J., Hadlock, T., Gent, A. and Strichartz, G.R. (2007) Tetracaine-Membrane Interactions: Effects of Lipid Composition and Phase on Drug Partitioning, Location and Ionization. Biophysical Journal, 92, 3988-4001.

https://doi.org/10.1529/biophysj.106.102434

[30] Strichartz, G.R., Sanchez, V., Arthur, G.R., Chaftez, R. and Martin, D. (1990) Fundamental Properties of Local Anesthetics. II. Measured Octanol: Buffer Partition Coefficients and pKa Values of Clinically-Used Drugs. Anesthesia \& Analgesia, 71, 158-170. https://doi.org/10.1213/00000539-199008000-00008

[31] Clement, N.R. and Gould, J.M. (1981) Pyranine (8-Hydroxy-1,3,6-pyrenetrisulfonate) as a Probe of Internal Aqueous Hydrogen Ion Concentration in Phospholipid Vesicles. Biochemistry, 20, 1534-1538. https://doi.org/10.1021/bi00509a019

[32] Hope, M., Bally, M., Webb, G. and Cullis, P. (1985) Production of Large Unilamellar Vesicles by a Rapid Extrusion Technique. Biochimica et Biophysica Acta, 812, 55-65. https://doi.org/10.1016/0005-2736(85)90521-8

[33] Damiano, E., Bassilana, M., Rigaud, J.L. and Leblanc, G. (1984) Use of the pH Sensitive Fluorescence Probe Pyranine to Monitor Internal pH Changes in Escherichia coli Membrane Vesicles. FEBS Letters, 166, 120-124. https://doi.org/10.1016/0014-5793(84)80056-3

[34] Kamp, F. and Hamilton, J.A. (1992) pH Gradient across Phospholipid Membranes Caused by Fast Flip-Flop of Un-Ionized Fatty Acids. Proceedings of the National 
Academy of Sciences of the United States of America, 89, 11367-11370.

https://doi.org/10.1073/pnas.89.23.11367

[35] Yeagle, P.L. (1985) Cholesterol and the Cell Membrane. Biochimica et Biophysica Acta. Reviews on Biomembranes, 822, 267-287. https://doi.org/10.1016/0304-4157(85)90011-5

[36] Schroeder, F., Woodford, J.K., et al. (1995) Cholesterol Domains in Biological Membranes. Molecular Membrane Biology, 12, 113-119. https://doi.org/10.3109/09687689509038505

[37] Ohvo-Rekilä, H., Ramstedt, B., Leppimäki, P. and Slotte, J. (2002) Cholesterol Interactions with Phospholipids in Membranes. Progress in Lipid Research, 41, 66-97. https://doi.org/10.1016/S0163-7827(01)00020-0

[38] Hung, W.C., Lee, M.T., Chen, F.Y. and Huang, H.W. (2007) The Condensing Effect of Cholesterol in Lipid Bilayers. Biophysical Journal, 92, 3960-3967. https://doi.org/10.1529/biophysj.106.099234

[39] de Meyer, F. and Smit, B. (2009) Effect of Cholesterol on the Structure of a Phospholipid Bilayer. Proceedings of the National Academy of Sciences of the United States of America, 106, 3654-3658. https://doi.org/10.1073/pnas.0809959106

[40] Auger, M., Smith, I.C. and Jarrell, H.C. (1989) Interactions of the Local Anesthetic Tetracaine with Glyceroglycolipid Bilayers: A ${ }^{2} \mathrm{H}-\mathrm{NMR}$ Study. Biochimica et Biophysica Acta, 981, 351-357. https://doi.org/10.1016/0005-2736(89)90047-3

[41] Bartucci, R., Mollica, P., Sapia, P. and Sportelli, L. (1998) Procaine Interaction with DPPC Multilayers: An ESR Spin Label Investigation. Applied Magnetic Resonance, 15, 181-195. https://doi.org/10.1007/BF03161870

[42] Boulanger, Y., Schreier, S., Leitch, L. and Smith, I. (1980) Multiple Binding Sites for Local Anesthetics in Membranes: Characterization of the Sites and Their Equilibria by Deuterium NMR of Specifically Deuterated Procaine and Tetracaine. Canadian Journal of Biochemistry, 58, 986-995. https://doi.org/10.1139/o80-134

[43] Boulanger, Y., Schreier, S. and Smith, I. (1981) Molecular Details of the Anesthetic-Lipid Interaction as Seen by Deuterium and Phophorous-31 NMR. Biochemistry, 20, 6824-6830. https://doi.org/10.1021/bi00527a013

[44] Kelusky, E.C. and Smith, I.C. (1984) The Influence of Local Anesthetics on Molecular Organization in Phosphatidylethanolamine Membranes. Molecular Pharmacology, 26, 314-321.

[45] Kuroda, Y. and Fujiwara, Y. (1987) Locations and Dynamical Perturbations for Lipids of Cationic Forms of Procaine, Tetracaine, and Dibucaine in Small Unilamellar Phosphatidylcholine Vesicles as Studied by Nuclear Overhauser Effects in $1 \mathrm{H} \mathrm{Nuc-}$ lear Magnetic Resonance Spectroscopy. Biochimica et Biophysica Acta, 903, 395-410. https://doi.org/10.1016/0005-2736(87)90046-0

[46] Papahadjopoulos, D., Jacobson, K., Poste, G. and Shepherd, G. (1975) Effects of Local Anesthetics on Membrane Properties. I. Changes in the Fluidity of Phospholipid Bilayers. Biochimica et Biophysica Acta, 394, 504-519. https://doi.org/10.1016/0005-2736(75)90137-6

[47] Ondrias, K., Horvath, L.I., Balgavy, P. and Stolc, S. (1984) Effects of Tertiary Amine Local Anaesthetics on the Phase Behaviour of the Dipalmitoylphosphatidylcholine Model Membrane. Electron Spin Resonance Tetrametylpiperidinyloxyl Partition Study. Physiol Bohemoslov, 33, 489-494.

[48] Kelusky, E.C., Boulanger, Y., Schreier, S. and Smith, I.C. (1986) An 2H-NMR Study of the Interactions of the Local Anesthetic Tetracaine with Membranes Containing Phosphatidyl Serine. Biochimica et Biophysica Acta, 856, 85-90. 
https://doi.org/10.1016/0005-2736(86)90013-1

[49] McLaughlin, S. (1977) Electrostatic Potentials at Membrane-Solution Interfaces. In: Bronnen, F. and Kleinzellen, A., Eds., Current Topics in Membranes and Transport, Academic Press, Cambridge, Vol. 9, 71-144.

https://doi.org/10.1016/S0070-2161(08)60677-2

[50] Westman, J., Boulanger, Y., Ehrenberg, A. and Smith, I. (1982) Charge and pH Dependent Drug Binding to Model Membranes. A ${ }^{2} \mathrm{H}-\mathrm{NMR}$ and Light Adsorption Study. Biochimica et Biophysica Acta, 685, 315-328.

https://doi.org/10.1016/0005-2736(82)90073-6 